\title{
Cathodoluminescence characterization of rare earth doped composite materials based on porous $\mathrm{GaP}$
}

\author{
B. Sánchez $\cdot$ B. Méndez $\cdot$ J. Piqueras $\cdot$ \\ L. Sirbu · I. M. Tiginyanu · V. V. Ursaki
}

Received: 8 June 2007/ Accepted: 12 September 2007/Published online: 17 October 2007

(C) Springer Science+Business Media, LLC 2007

\begin{abstract}
Porous GaP layers doped with erbium or europium elements have been obtained by electrochemical etching and further impregnation processes. The thermal treatments for optical activation of rare earth (RE) ions lead to partial oxidation of porous $\mathrm{GaP}$ skeleton and a composite material is obtained. The presence of $\mathrm{ErPO}_{4}$ and $\mathrm{EuPO}_{4}$ oxide nanophases is detected by X-ray diffraction (XRD) analysis. Visible luminescence from $\mathrm{RE}$ ions in the composite material has been investigated by means of the cathodoluminescence (CL) technique in the scanning electron microscope. Intense red and green emission lines characteristic from $\mathrm{Er}^{3+}$ and $\mathrm{Eu}^{3+}$ ions dominate the CL spectra in the case of parallel and regular nanotubes in the samples. The role of the oxygen content and the detected phases in the luminescence results are discussed.
\end{abstract}

\section{Introduction}

The growth of semiconductor nanostructures containing rare earth (RE) elements is of interest due to their

B. Sánchez · B. Méndez $(\bowtie) \cdot J$. Piqueras

Departamento Física de Materiales, Facultad de Ciencias Físicas,

Universidad Complutense de Madrid, 28040 Madrid, Spain

e-mail: bianchi@fis.ucm.es

L. Sirbu · I. M. Tiginyanu

National Center for Materials Study and Testing, Technical

University of Moldova, Chisinau, Republic of Moldova

I. M. Tiginyanu · V. V. Ursaki

Laboratory of Low-Dimensional Semiconductor Structures, Institute of Applied Physics, Academy of Sciences of Moldova, Chisinau, Republic of Moldova potential applications in optical nanodevices. Anodization is a relatively simple and cost-effective technique to obtain large area, porous semiconductor structures. Since the observed room temperature luminescence in porous silicon [1], a high amount of work has been devoted to study not only silicon, but also other porous semiconductors, such as III-V compounds [2]. In particular, previous works on porous $\mathrm{GaP}$ have revealed a sharp increase of the intensity of photoluminescence (PL) along with the emergence of blue and ultraviolet emission bands from the porous GaP layer [3-4], as compared with bulk material. This behavior is opposite to other porous III-V semiconductors such as InP or GaAs, in which the luminescence is quenched after the anodization process. Besides, the morphology of the porous layer may influence the relative intensities in the PL or cathodoluminescence (CL) bands in porous $\mathrm{GaP}$, as it has been previously reported [5]. RE ions embedded in different hosts have been used to achieve higher quantum efficiency from semiconductors, such as silicon, InP, or GaN. In the case of silicon, erbium ions have been embedded in nanocrystalline and amorphous thin films in order to enhance luminescence in the visible range [6]. The narrow emission lines due to intraionic recombinations of the $\mathrm{RE}$ ion have been shown to be almost independent of the host matrix. However, the intensity of the luminescence decreases as the temperature increases. This effect seems to be less noticeable in wide band gap semiconductors [7] and for this reason, wide band gap III-V semiconductors, e.g., $\mathrm{GaN}$, or semiconductor oxides, e.g., $\mathrm{Ga}_{2} \mathrm{O}_{3}$, have been recently used to host $\mathrm{RE}$ ions such as $\mathrm{Er}^{3+}$, $\mathrm{Yb}^{3+}$, or $\mathrm{Eu}^{3+}$ [8-9]. In this work, we investigate the luminescence properties of $\mathrm{Er}$ and Eu doped composite materials prepared on porous GaP layers with different morphologies. 


\section{Experimental}

The samples investigated in this work have been obtained by anodization of n-type $\mathrm{GaP}$ wafers under different conditions leading to two specific porous morphologies, which we will refer as $\mathrm{H}$ and $\mathrm{T}$. Morphology $\mathrm{H}$ consists of irregular "holes" of variable sizes with random spatial distribution, while morphology $\mathrm{T}$ is characterized by the presence of regular "tubes" or pores. RE ions were incorporated into both kinds of porous layers from $\mathrm{EuCl}_{3}: \mathrm{C}_{2} \mathrm{H}_{5} \mathrm{OH}$ and $\mathrm{ErCl}_{3}: \mathrm{C}_{2} \mathrm{H}_{5} \mathrm{OH}$ solutions. Afterwards, the samples were annealed using a halogen lamp heater at $800{ }^{\circ} \mathrm{C}$ for $20 \mathrm{~min}$ as described elsewhere [10].

Cathodoluminescence measurements were performed in the visible range at $90 \mathrm{~K}$ using an accelerating voltage of $20 \mathrm{kV}$ in a Leica 440 or Hitachi S-2500 scanning electron microscopes (SEM). CL spectra were acquired with a Hamamatsu PMA-11 charge-couple-device. Energy dispersive X-ray microanalysis has also been used to assess the composition of the produced materials and the incorporation of $\mathrm{Eu}$ or Er into the samples. In addition, X-ray diffraction (XRD) analysis of the samples was performed with a Philips X-Pert MPD System with $\mathrm{Cu} \mathrm{K}_{\alpha 1}$ radiation.

\section{Results and discussion}

Figure 1 shows SEM micrographs taken in cross-section from the RE doped samples prepared on porous GaP layers. Fig. 1a shows a representative SEM micrograph of the Er doped layer with $\mathrm{H}$ morphology with submicron holes as well as holes with larger transverse dimensions. A SEM image of a layer with T morphology is shown in Fig. 1b. In this case, the porous structure consists of parallel tubes of sub micrometer diameter, which eventually develops a tree-like structure, with secondary tubes emerging from the main branch. In both morphologies, the CL intensity is higher in the porous layer than in the bulk substrate and the spectral analysis exhibits differences between the $\mathrm{H}$ and the $\mathrm{T}$ structures.

In order to study the characteristics of the CL emission of different samples, an undoped porous GaP layer was also analyzed for comparison. The CL spectrum of the undoped porous layer is shown in Fig. 2a. The main emission band is normally referred as red band in $\mathrm{GaP}$, centered at about $1.72 \mathrm{eV}$, which is associated with a deep donor-acceptor defect center [11] while the indirect near band edge emission at $2.23 \mathrm{eV}$ is not observed.

The CL spectrum of an Er doped sample with $\mathrm{H}$ morphology is shown in Fig. 2b. Along with the red defect band at $1.68 \mathrm{eV}$ of $\mathrm{GaP}$, narrower and weaker peaks at $1.85,1.88,2.25$, and $2.27 \mathrm{eV}$ are also resolved. These emissions correspond to red and green intraionic emission lines from $\mathrm{Er}^{3+}$ ions. From these observations, we can conclude that $\mathrm{H}$ morphology enables the detection of the RE emission lines, but the dominant emission band corresponds with defects in the porous GaP matrix.

Let us now consider Er and Eu doped samples with T morphology. The CL spectrum from the Er doped layer is shown in Fig. 3a. In this case, the layer consists of small interconnected pieces of tubes. Two red $(1.85$ and $1.88 \mathrm{eV})$ and two green $(2.25$ and $2.27 \mathrm{eV})$ emission lines from the Er ions are very well resolved and the green emission dominates the CL spectrum. In the case of Eu doped layers, the corresponding CL spectrum (Fig. 3b) shows a high signal level and the intraionic $\mathrm{Eu}^{3+}$ emission lines are clearly resolved in the green region, between 2.01 and $2.11 \mathrm{eV}$. In addition, two narrow red peaks at 1.81 and $1.77 \mathrm{eV}$, close to the defect $\mathrm{GaP}$ band, and a rather intense and broad blue-UV band (not shown) are detected. Sharp CL peaks at the same positions $(1.81$ and $1.71 \mathrm{eV})$ have been found in gallium oxide nano- and microwires obtained after a vapor solid process [12]. On the other hand, the blue-UV band is usually attributed to oxygen vacancies in $\mathrm{Ga}_{2} \mathrm{O}_{3}$ [13]. Therefore, our results suggest that gallium oxide has been formed during the thermal treatment at $800{ }^{\circ} \mathrm{C}$ used to optical activation of $\mathrm{Eu}^{3+}$ ions.

The obtained CL results show that the nanotube array structure, either in Er or Eu doped samples, leads to higher quantum efficiency of RE related luminescence. The $T$ morphology proves to be more suitable to get luminescence from RE ions than morphology H. Narrow red and green peaks are clearly resolved and become the prevalent emission lines.
Fig. 1 Representative SEM micrographs showing crosssectional views: (a) morphology of holes " $\mathrm{H}$ " and (b) morphology of tubes "T"
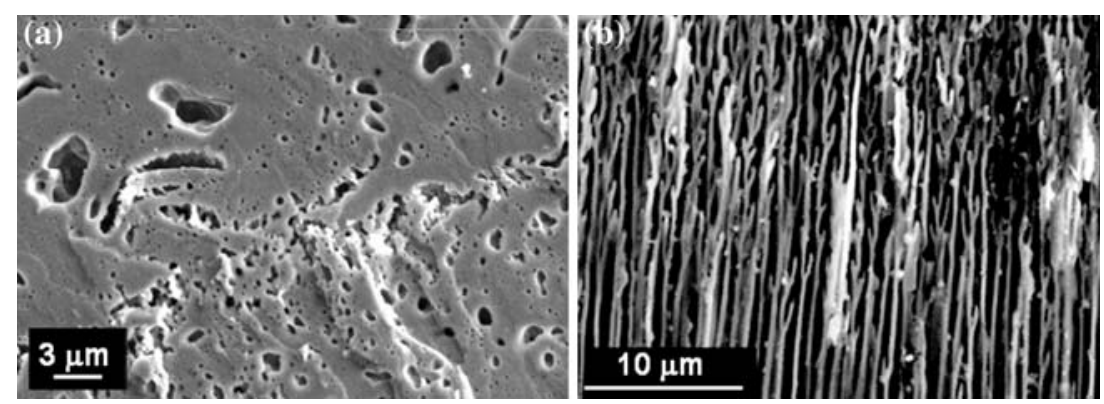

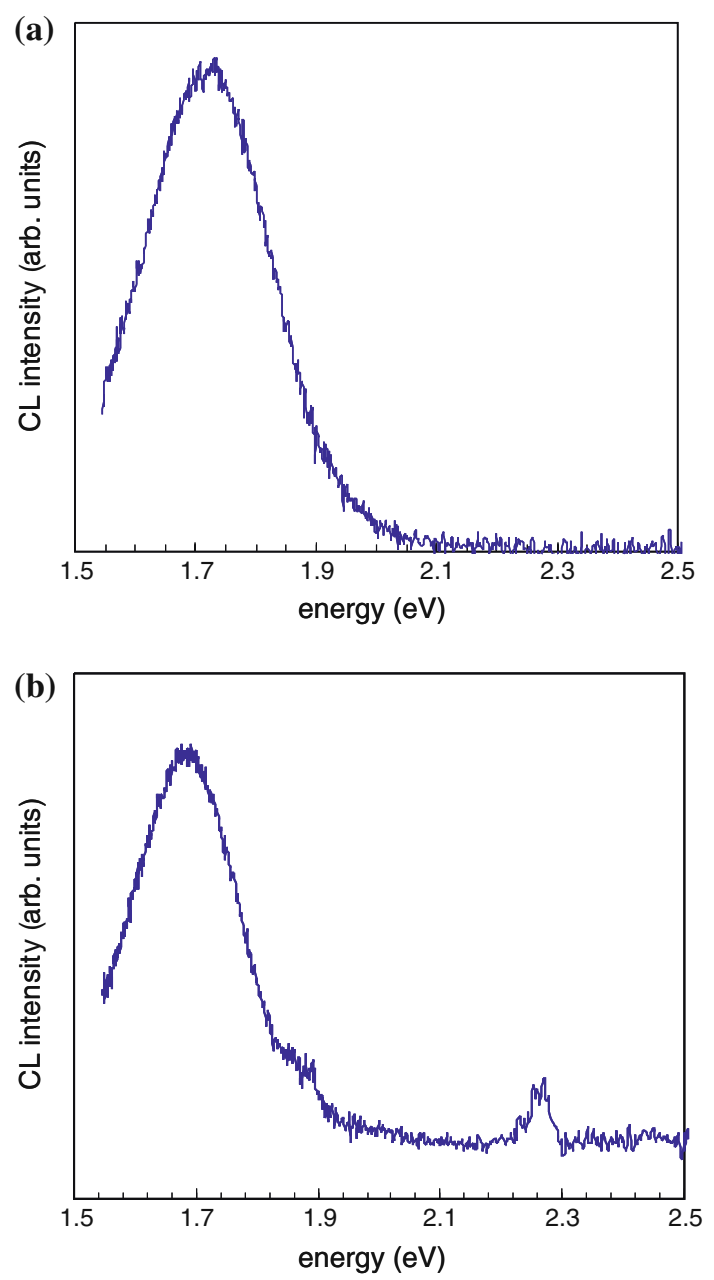

Fig. 2 (a) CL spectrum of the undoped porous GaP layer; (b) CL spectra from a sample with morphology $\mathrm{H}$ doped with erbium

It was demonstrated that the presence of oxygen is imperative for achieving efficient emission from RE ions introduced into silicon [14] or III-V material [15]. The oxygen co-doping leads to the formation of quasi-molecular centers at low impurity density [16] and to the segregation of an oxide phase at higher doping levels [17] in zinc blende compounds. We have performed energy dispersive $\mathrm{X}$-ray microanalysis and the results are displayed in Table 1. Er and Eu percentages reach values of 3-4\% in layers with $\mathrm{H}$ morphology, which explains the presence of weak RE emission lines in the corresponding CL spectra shown in Fig. 2b. On the other hand, in samples with $\mathrm{T}$ morphology the percentage of the RE element is around $10 \%$ for both RE dopants. The results agree with the relative intense $\mathrm{RE}$ emission lines observed in the $\mathrm{CL}$ spectra (Fig. 3). It should be noticed that a considerable amount of oxygen is also measured in the last set. Therefore, the intrinsic high surface-volume ratio of porous structures enables the incorporation of a big amount of RE by impregnation, especially in the case of $\mathrm{T}$ morphology.
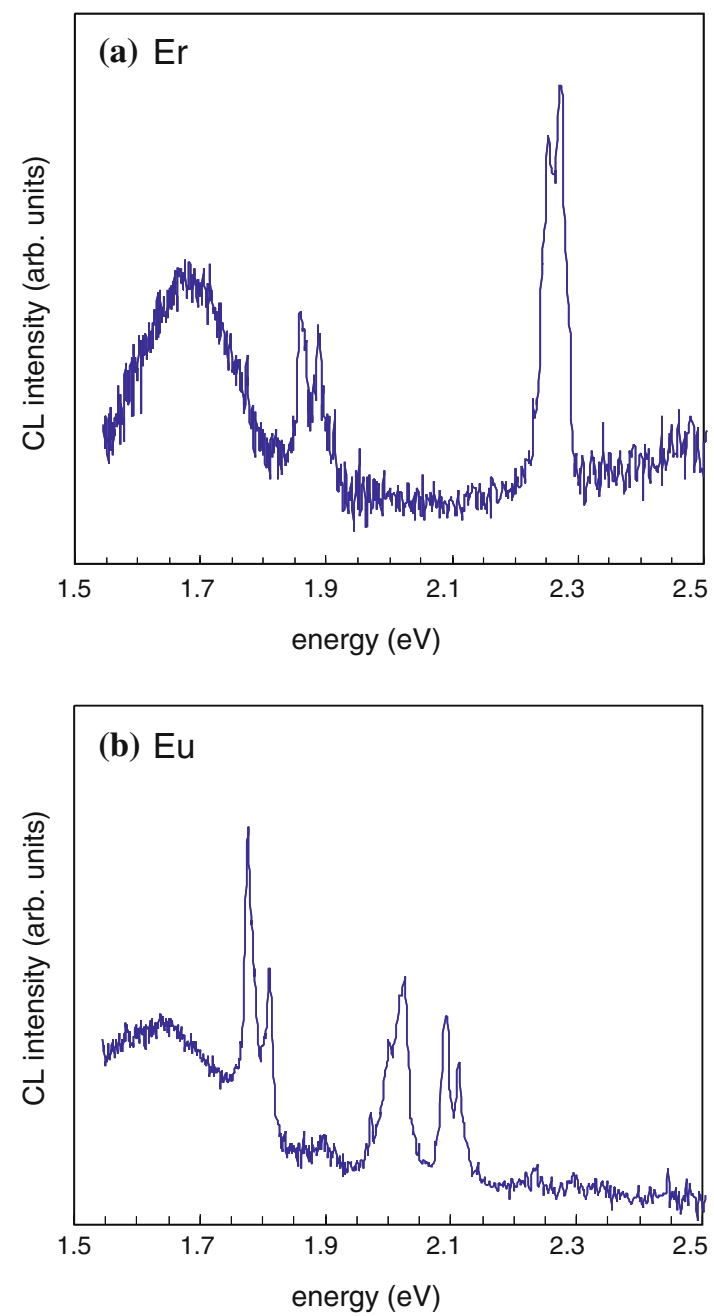

Fig. 3 CL spectra from the nanotubes array (morphology T) doped with (a) erbium and (b) europium

On the other hand, the high temperature treatment seems to lead to the oxidation of a considerable part of the porous $\mathrm{GaP}$ skeleton, i.e., specific composite materials are produced.

In order to identify the phases responsible for the emission related to intrashell transitions in RE ions, XRD analysis of the composites were performed. The XRD results demonstrate the formation of $\mathrm{a} \mathrm{CaO}_{4}$ phase with the low-cristobalite (orthorhombic $\mathrm{C} 222_{1}$ ) structure as well the $\mathrm{ErPO}_{4}$ or $\mathrm{EuPO}_{4} \mathrm{RE}$ related oxides (Fig. 4). The

Table 1 Summary of X-ray microanalysis data

\begin{tabular}{llllll}
\hline Sample & $\begin{array}{l}\text { Ga } \\
\text { (\% weight) }\end{array}$ & $\begin{array}{l}\text { P } \\
(\% \text { weight })\end{array}$ & $\begin{array}{l}\text { O } \\
(\% \text { weight })\end{array}$ & $\begin{array}{l}\text { Er } \\
(\% \text { weight })\end{array}$ & $\begin{array}{l}\text { Eu } \\
(\% \text { weight })\end{array}$ \\
\hline Er-H & 57.35 & 25.86 & 1.82 & 4.65 & \\
Eu-H & 57.85 & 23.73 & 4.68 & & 2.99 \\
Er-T & 48.39 & 21.30 & 9.55 & 9.66 & \\
Eu-T & 49.14 & 19.48 & 5.27 & & 10.21 \\
\hline
\end{tabular}




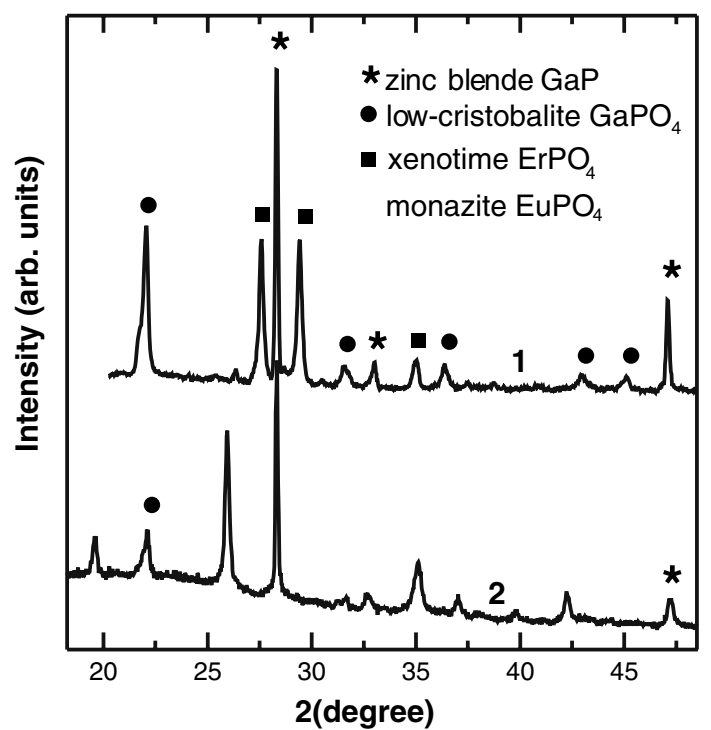

Fig. 4 XRD analysis of composites prepared on GaP templates doped with Er (curve 1) and Eu (curve 2)

rare-earth related phases were identified as tetragonal $\mathrm{I}_{1} /$ amd xenotime $\mathrm{ErPO}_{4}$ and monoclinic $\mathrm{P} 2_{1} / n$ monazite $\mathrm{EuPO}_{4}$. The coordination number for rare-earth ions in $\mathrm{ErPO}_{4}$ and $\mathrm{EuPO}_{4}$ is eight and nine, respectively. It is known that in both the $\mathrm{GaP}$ host and $\mathrm{GaPO}_{4}$ the $\mathrm{Ga}$ atoms are tetrahedral coordinated, while the RE ions prefer a coordination number higher than six. Therefore the segregation of the RE impurities is more favorable in the form of finely dispersed $\mathrm{ErPO}_{4}$ and $\mathrm{EuPO}_{4}$ nanophases in the composite, rather than their incorporation into the $\mathrm{GaP}$ or $\mathrm{GaPO}_{4}$ host.

\section{Conclusions}

Composite materials doped with $\mathrm{Er}$ and $\mathrm{Eu}$ have been fabricated by a simple impregnation of RE ions from solutions into porous $\mathrm{GaP}$ layers prepared by electrochemical etching process and characterized by means of the CL technique. The results show that porous layers with parallel and regular nanotubes represent a more efficient host than holes of irregular shape to achieve luminescence from RE ions, and that this emission is stable after long time storage. This applies to both $\mathrm{Er}$ and Eu doped samples. Thermal treatments at $800{ }^{\circ} \mathrm{C}$ lead to a partial oxidation of the $\mathrm{GaP}$ skeleton and the formation of a $\mathrm{GaPO}_{4}$ native oxide with low-cristobalite structure. The produced composite materials exhibit efficient green and red emission that comes from finely dispersed $\mathrm{ErPO}_{4}$ and $\mathrm{EuPO}_{4}$ oxide nanophases in the composite.

Acknowledgement This work has been supported by MEC (project MAT-2003-00455).

\section{References}

1. Canham LT (1990) Appl Phys Lett 57:1046

2. Föll H, Langa S, Carstensen J, Christophersen M, Tiginyanu IM (2003) Adv Mater 15:183

3. Anedda A, Serpi A, Karavanskii VA, Tiginyanu IM, Ichizli VM (1995) Appl Phys Lett 67:3316

4. Kuriyama K, Ushiyama K, Ohbora K, Miyamoto Y, Takeda S (1998) Phys Rev B 58:1103

5. Stevens-Kalceff M, Tiginyanu IM, Langa S, Föll H (2001) J Appl Phys 89:2560

6. Nogales E, Méndez B, Piqueras J, Plugaru R, Coraci A, García JA (2002) J Phys D 35:295

7. Favennec PN, Haridon HL, Salvi M, Muotonnet D, Le Guillo Y (1989) Electron Lett 25:718

8. Nogales E, Martin RW, O'Donnell KP, Lorenz K, Alves E, Ruffenach S, Briot O (2006) Appl Phys Lett 88:031902

9. Gollakota P, Dhawan A, Wellenius P, Lunardi LM, Muth JF, Saripalli YN, Peng HY, Everitt HO (2006) Appl Phys Lett 88:221906

10. Sirbu L, Ursaki VV, Tiginyanu IM, Dolgaleva K, Boyd RW (2007) Phys Stat Sol (RRL) 1:R13

11. Domínguez-Adame F, Piqueras J (1989) Mater Chem Phys 21:539

12. Nogales E, Méndez B, Piqueras J (2005) Appl Phys Lett 86:113112

13. Villora EG, Atou T, Sekiguchi T, Sugawara T, Kikuchi M, Fukuda T (2001) Solid State Commun 120:455

14. Nogales E, Méndez B, Piqueras J, Plugaru R, Coraci A, García JA (2002) J Phys D: Appl Phys 35:295

15. Colon JE, Elsaesser DW, Yeo YK, Hengehold RL, Pomrenke GS (1993) Appl Phys Lett 63:216

16. Yoshida M, Hiraka K, Ohta H, Fujiwara Y, Koizumi A, Takeda Y (2004) J Appl Phys 96:4189

17. Phillips JC (1994) J Appl Phys 76:5896 